\title{
BilKristal 2.0: A tool for pattern information extraction from crystal structures
}

\author{
Erhan Okuyan, Uğur Güdükbay* \\ Department of Computer Engineering, Bilkent University, 06800, Ankara, Turkey
}

\section{A R T I C L E I N F O}

\section{Article history:}

Received 26 August 2013

Accepted 23 September 2013

Available online 5 October 2013

\section{Keywords:}

Crystal

Crystallography

Material science

Pattern recognition

Primitive vectors

Basis vectors

Space group

Symmetry

\begin{abstract}
A B S T R A C T
We present a revised version of the BilKristal tool of Okuyan et al. (2007). We converted the development environment into Microsoft Visual Studio 2005 in order to resolve compatibility issues. We added multicore CPU support and improvements are made to graphics functions in order to improve performance. Discovered bugs are fixed and exporting functionality to a material visualization tool is added.
\end{abstract}

\section{New version program summary}

Program title: BilKristal 2.0.

Catalogue identifier: ADYU_v2_0

Program summary URL: http://cpc.cs.qub.ac.uk/summaries/ADYU_v2_0.html

Program obtainable from: CPC Program Library, Queen's University, Belfast, N. Ireland

Licensing provisions: Standard CPC licence, http://cpc.cs.qub.ac.uk/licence/licence.html

No. of lines in distributed program, including test data, etc.: 364263

No. of bytes in distributed program, including test data, etc.: 9135815

Distribution format: tar.gz

Programming language: C, C++, Microsoft.NET Framework 2.0 and OpenGL Libraries.

Computer: Personal computer with Windows operating system.

Operating system: Windows XP or higher.

Has the code been vectorized or parallelized?: Multi-core CPU support included.

RAM: 20-60 Megabytes.

Catalogue identifier of previous version: ADYU_v1_0

Journal reference of previous version: Comput. Phys. Comm. 176 (2007) 486

Classification: 8.

External routines: Microsoft.NET Framework 2.0. For the visualization tool, the graphics card driver should also support OpenGL.

Does the new version supercede the previous version?: Yes

Nature of problem:

Determining the crystal structure parameters of a material is a very important issue in crystallography. Knowing the crystal structure parameters helps the understanding of the physical behavior of a material. For complex structures, particularly for materials which also contain local symmetry as well as global symmetry, obtaining crystal parameters can be very hard. Solution method:

The tool extracts crystal parameters such as primitive vectors and basis vectors and identifies the space group from the atomic coordinates of crystal structures.

Reasons for new version:

Additional features, resolved compatibility issues with the new development environments, performance optimizations, minor bug corrections.

Summary of revisions:

- Capability to export to MaterialVis tool [1] is added. The tool can export the unit cell information extracted from the crystal structure, the raw atomic coordinates and atomic radii into a data file (.dat) that the MaterialVis tool can process.

\footnotetext{
* Corresponding author. Tel.: +90 312290 1386; fax: +90 3122664047.

E-mail address: gudukbay@cs.bilkent.edu.tr (U. Güdükbay).
} 
- Compatibility issues with Microsoft Visual Studio 2005 up to 2010 are resolved. The original code was developed using Microsoft Visual Studio 2003. However, newer Visual Studio versions were not able to convert and compile the code. Due to the changes in the .NET framework, the converted project produced many errors. In this work, the project is converted into a Visual Studio 2005 project and compilation errors are resolved. We also tested the code with Visual Studio 2010 and the project was successfully converted and compiled.

- Multi-Core CPU support is added. In recent years, multi-core CPUs have become very common. We added the multi-core CPU support in order to utilize the computational capabilities of additional CPU cores. This significantly improves the performance.

- The visualization interface is improved. In particular, the sphere drawing functionality is replaced with an efficient and high quality version that utilizes GPU acceleration.

- For some cases, the fractional coordinates of some of the calculated basis vectors were not all in the $[0,1)$ range, but at coordinate 1.0 for some axes. These cases were corrected by translating these basis vectors into the $[0,1)$ range.

Restrictions:

Assumptions are explained in [2]. However, none of them can be considered as a restriction on the complexity of the problem.

Running time:

The tool was able to process input files with more than a million atoms in less than $20 \mathrm{~s}$ on a PC with an Athlon quad-core $\mathrm{CPU}$ at $3.2 \mathrm{GHz}$ using the default parameter values.

References:

[1] Erhan Okuyan, Ugur Güdükbay, MaterialVis: Crystal and Amorphous Material Visualization Tool Using Direct Volume and Surface Rendering Techniques (Program Summary), Computer Physics Communications, Submitted.

[2] Erhan Okuyan, Ugur Güdükbay, and Oguz Gülseren, Pattern Information Extraction from Crystal Structures, Computer Physics Communications, 176 (2007) 486.

(C) 2013 Elsevier B.V. All rights reserved. 